\title{
Poliquetos (Annelida: Polychaeta) epibiontes de Spondylus americanus (Bivalvia: Spondylidae) en el Parque Nacional Mochima, Venezuela
}

\author{
Ildefonso Liñero Arana \& Oscar Díaz Díaz \\ Instituto Oceanográfico de Venezuela, Edif. I.O.V. piso 2, labs. 208-210. Av. Universidad. Cerro Colorado, Universidad \\ de Oriente, Venezuela; ilinero@cantv.net; ecobentos12@hotmail.com; oscarfelipediazd@yahoo.es
}

Recibido 04-VIII-2004. C Corregido 08-IX-2005. Aceptado 09-III-2006.

\begin{abstract}
Polychaetes (Annelida: Polychaeta) epibiont on Spondylus americanus (Bivalvia: Spondylidae) from Mochima National Park, Venezuela. The polychaetes epibiontic on the mollusk Spondylus americanus Hermann, 1781 were extracted from mollusks hand-collected at a depth of 10-30 m in Mochima National Park, Venezuela $\left(10^{\circ} 21^{\prime} 00^{\prime \prime} \mathrm{N}-63^{\circ} 23^{\prime} 36^{\prime} \mathrm{W}\right)$, using scuba diving gear. Forty-three polychaete species were identified on the 32 bivalve specimens analyzed. The Serpulidae included 17 especies, Eunicidae six and Terebellidae four species. The most abundant species were Hydroides dirampha Mörch, 1863, Pileolaria militaris Claparède, 1868 (Serpulidae), and Notaulax nudicollis Krøyer, 1856 (Sabellidae). Their geographic affinitie were: 51.3\% Atlantic, 28.2\% widely distributed, $17.9 \%$ Amphiamericans, and 2.6\% have a disjunct distribution. Rev. Biol. Trop. 54 (3): 765-772. Epub 2006 Sept. 29.
\end{abstract}

Key words: polychaetes, Spondylus americanus, Venezuela, bivalves.

El ostión espinoso americano, Spondylus americanus Hermann, 1781, es la mayor de las tres especies de espondílidos registradas para el Gran Caribe (Abbott 1974). Está principalmente adherida a rocas, corales y sustratos duros y se distribuye desde Carolina del Norte hasta Brasil (Humfrey 1975, Díaz y Puyana 1994). De valvas gruesas y fuertes presenta la superficie recubierta por espinas largas y delgadas, y otras cortas. Esta característica proporciona refugio y protección a la fauna móvil, mientras que la disponibilidad de una mayor cantidad de microhábitats permite su colonización por la fauna sedentaria o sésil, entre la que se cuentan moluscos, poríferos, cnidarios, tunicados, crustáceos y poliquetos. Este último grupo constituye uno de los más importantes debido a su abundancia, diversidad, capacidad de colonización y distribución. Algunas especies de espiónidos, principalmente, y cirratúlidos y serpúlidos en menor incidencia, han sido señalados en la literatura como responsables de mortandad, baja tasa de crecimiento y disminución del precio comercial de pectínidos, mejillones y ostras (Ciocco 1990, Basilio et al. 1995, Cáceres-Martínez 2001). Sin embargo, en general, los estudios sobre poliquetos epibiontes de moluscos son escasos. Para las costas del Pacífico americano, se ha trabajado con Pinna bicolor Gmelin, 1791 (Keough 1984), Spondylus princeps unicolor Sowerby, 1847 (De León-González et al. 1993) y Argopecten purpuratus Lamarck, 1819 (Basilio et al. 1995); mientras que para Venezuela se ha estudiado la epifauna de Pinctada imbricata Röding, 1798 (Díaz y Liñero-Arana 2003a), Isognomun alatus Gmelin, 1791 (Díaz y Liñero-Arana 2003b) y la fauna acompañante de Perna viridis (Linnaeus, 1758) (Liñero-Arana 1999).

En el presente trabajo se hace un estudio de la poliquetofauna epibionte del ostión espinoso americano S. americanus en la Bahía de Mochima, costa nororiental de Venezuela, así como el análisis biogeográfico de dicha taxocenosis. 


\section{MATERIALES Y MÉTODOS}

Área de estudio: Los ejemplares de $S$. americanus fueron colectados en el Parque Nacional Mochima (10²1'00" N - 63'23'36" W). Las recolectas se realizaron de forma manual entre 10 y $30 \mathrm{~m}$ empleando equipo de buceo autónomo. Los organismos fueron colocados en bolsas plásticas debidamente etiquetadas y éstas, a su vez, dentro de cavas con hielo y agua de mar para su traslado. Una vez en el laboratorio, los organismos fueron dispuestos en acuarios con agua de mar y bombas para aireación. Posteriormente, se procedió a separar los poliquetos epibiontes de la superficie de $S$. americanus y a fijarlos en una solución de formaldehído al $8 \%$ en agua de mar. Para la identificación de los poliquetos se procedió de acuerdo con la metodología descrita por Díaz y Liñero-Arana (2003a, b).

\section{RESULTADOS}

Se examinó un total de 32 ejemplares del bivalvo, recolectándose 569 ejemplares de poliquetos pertenecientes a 43 especies y 15 familias (Cuadro 1). La familia Serpulidae fue la mejor representada con 17 especies, seguida de Eunicidae con seis y Terebellidae con cuatro. Como especies más abundantes resultaron Hydroides dirampha Mörch, 1863, Pileolaria militaris Claparède, 1868 (Serpulidae), y Notaulax nudicollis Krøyer, 1856 (Sabellidae). Entre las especies identificadas, Sclerostyla ctenactis Mörch, 1863, Pseudovermilia holcopleura ten Hove, 1975 y Thelepus setosus (de Quatrefages, 1865), constituyen primeros registros para Venezuela. Hydroides gairacensis Augener, 1934 constituye el segundo registro para la costa de Venezuela desde que Mörch 1863 (Bastida-Zavala y ten Hove 2002) la citara para La Guaira.

\section{DISCUSIÓN}

El número de especies registrado en estudios similares es inferior al obtenido en éste.
Liñero-Arana (1999) registró 11 especies asociadas a Perna viridis; Díaz y Liñero-Arana (2003a, b) para P. imbricata e I. alatus informaron 26 y 38 especies, respectivamente; Keough (1984) identificó tres especies de espirórbidos en Pinna bicolor Gmelin, 1791. De León-González et al. (1993), registraron 31 especies de poliquetos epibiontes de Spondylus princeps unicolor Sowerby, 1847, en el Pacífico americano. En el presente estudio se registran 12 especies más, de las cuales ocho especies son comunes (Syllis (Syllis) gracilis, Eunice cariboea, E. vittata, Lysidice ninetta, Thelepus setosus. Pseudovermilia occidentalis, Hydroides gairacensis y Sclerostyla ctenactis). En ambos estudios la familia Serpulidae fue la mejor representada, tanto en número de especies como en abundancia, constituyendo en nuestro estudio el $39.5 \%$ de las especies y el $56,8 \%$ de la abundancia total. La dominancia de los serpúlidos obedece a que las conchas del bivalvo constituyen un sustrato apropiado para su colonización y construcción de tubos de carbonato de calcio construidos por los miembros de esta familia. Por otro lado, la presencia de terebélidos, sabélidos, cirratúlidos y flabeligéridos, sobre las valvas, especies que normalmente construyen sus galerías en el sedimento, a excepción del sabélido $N$. nudicollis que se encuentra asociado a sustratos duros, ya que puede horadar sustratos calcáreos (Perkins 1984, Liñero-Arana 1996, Díaz 1999), obedece a la acumulación de sedimento entre las espinas del molusco o entre los tubos de los mismos serpúlidos lo que permite la colonización de estos microhábitats. Sin embargo, el número de especies de terebélidos y sabélidos fue inferior al registrado por De León-González et al. (1993), posiblemente debido a que $S$. princeps unicolor coloniza sustratos arenosos y/o areno-fangosos; mientras que $S$. americanus está asociado, principalmente a sustratos duros, aunque ambas especies presentan tallas y distribución batimétrica similares (Keen 1971, Abbott 1974, Humfrey 1975, Díaz y Puyana 1994).

Respecto a la presencia de Polydora websteri Hartman, 1943, especie que junto a otros espiónidos, ha sido registrada como plaga en 


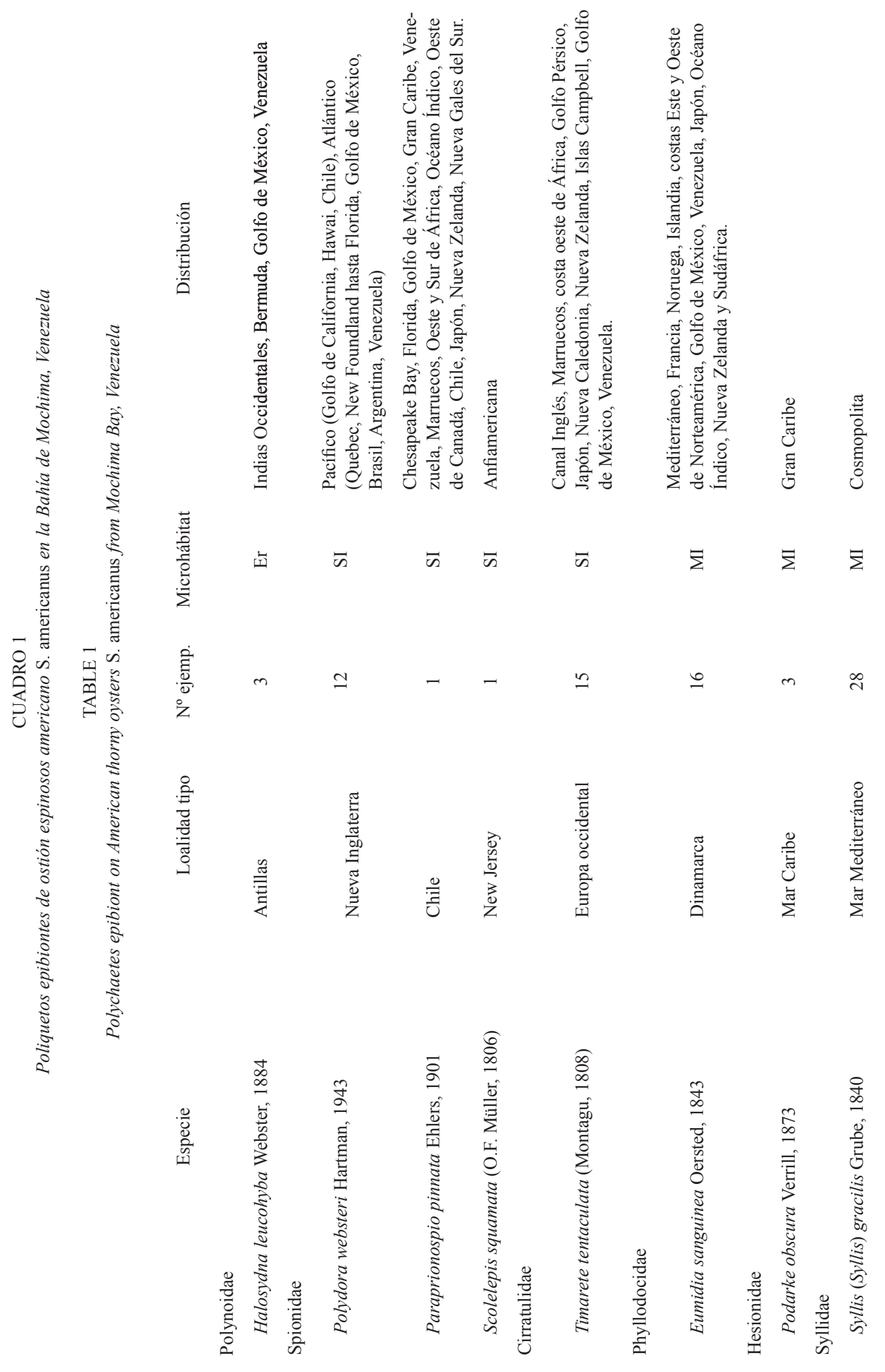




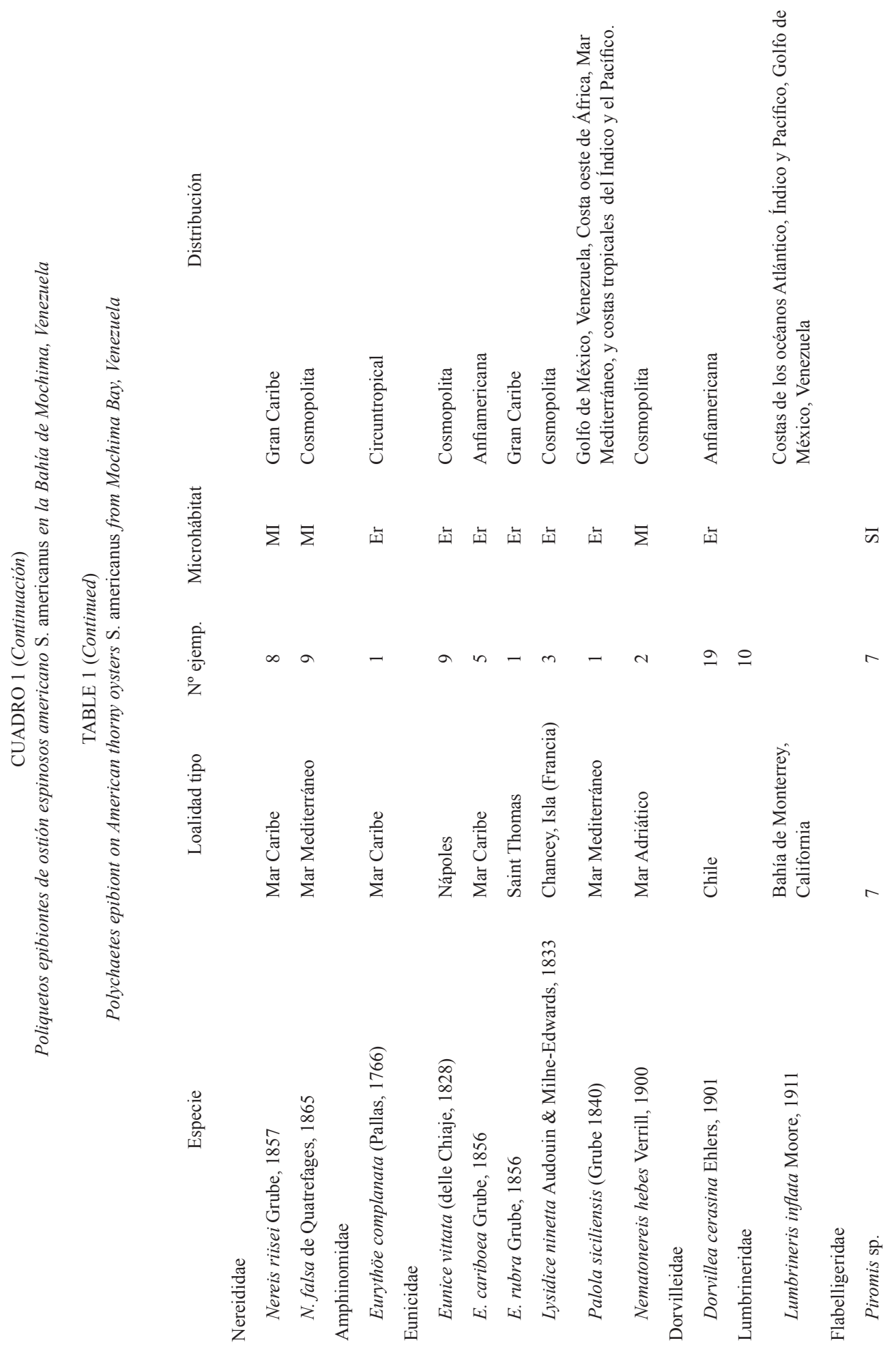




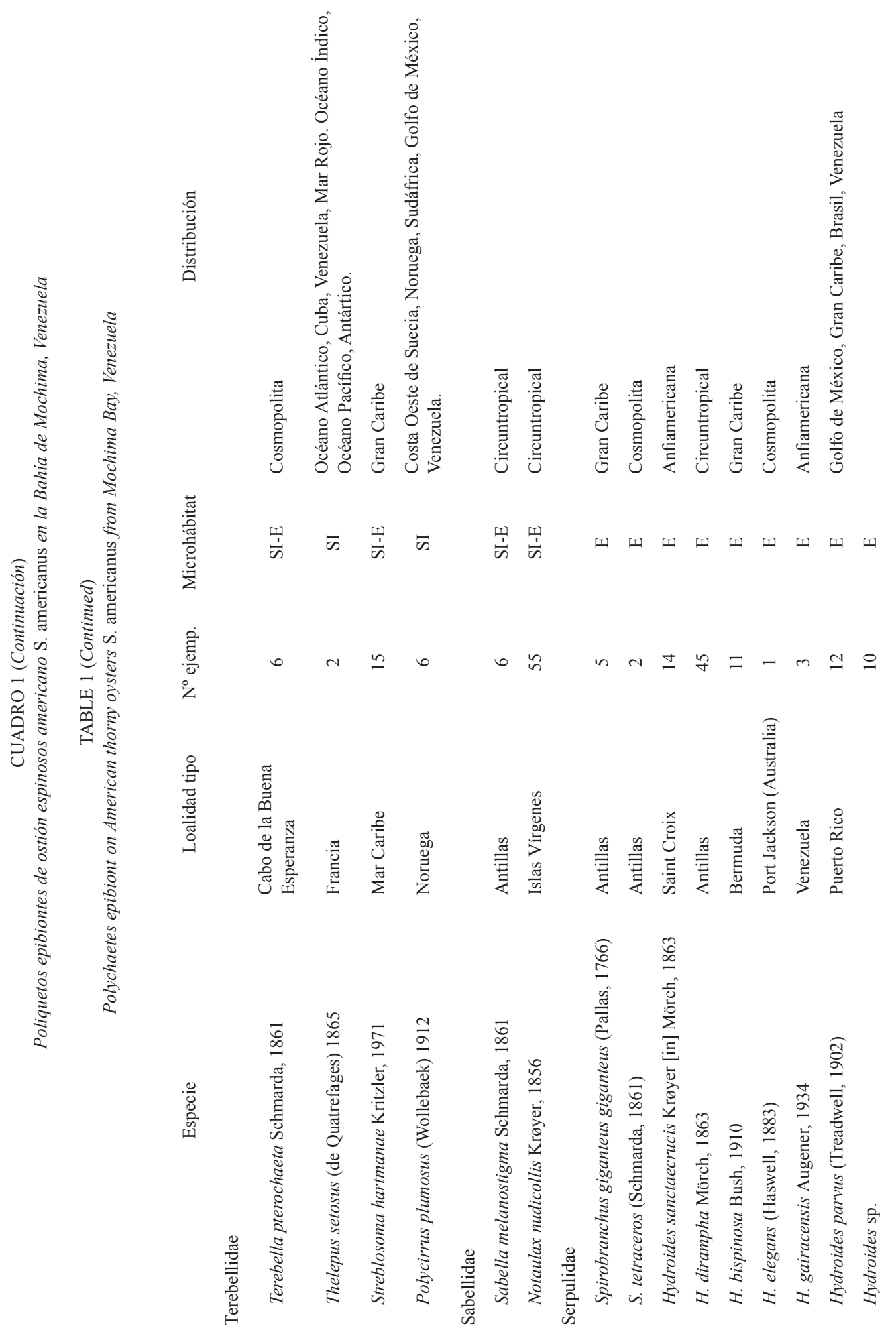




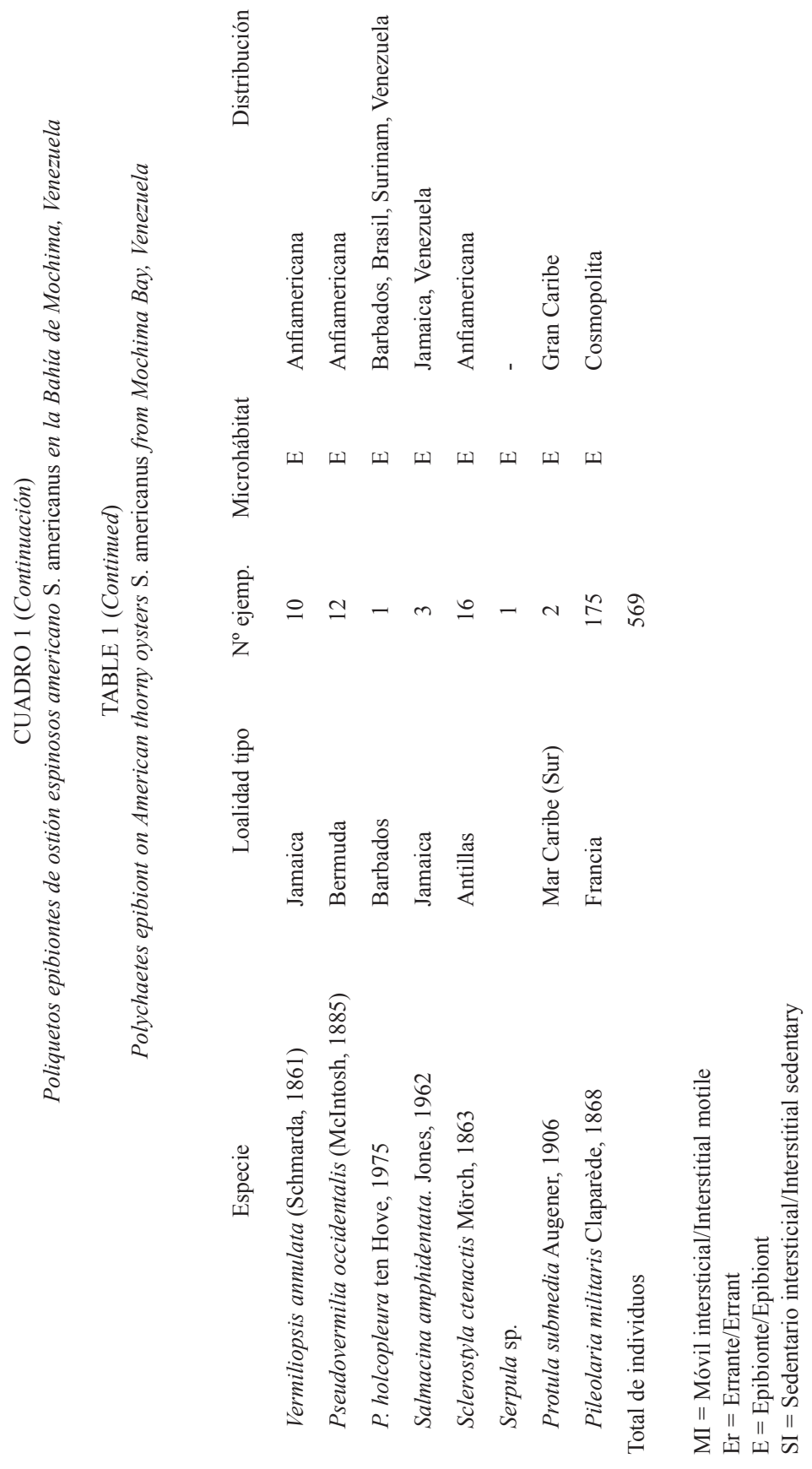


cultivos de bivalvos (Blake y Evans 1973, Widman y Rhodes 1991) su abundancia es considerablemente baja, apenas $2.1 \%$ del total.

La presencia de 28 especies de poliquetos sedentarios $(65.1 \%)$ evidencia la importancia del bivalvo como sustrato estable para el desarrollo de éstas, así como para el refugio de formas errantes, constituyendo así verdaderas islas ecológicas (De León González et al. 1993).

El estudio de la distribución geográfica (Cuadro 1) señala la presencia de 14 especies cosmopolitas (32.3\%), 10 especies del Atlántico (23.3\%), ocho anfiamericanas (18.6\%), cuatro circuntropicales $(9.3 \%)$, una registrada para los océanos Índico y Atlántico (2.3\%) y una disyunta (anfiamericana-transpacífica). En este análisis las especies predominantes corresponden al Atlántico tropical y subtropical (51.3\%), seguidas de las cosmopolitas (28.2\%). No se registraron especies endémicas. Este constituye el primer trabajo sobre poliquetos epibiontes de $S$. americanus en esta región del Atlántico. Es importante señalar que en el presente estudio se registraron especies que presentan una amplia distribución geográfica que, aun cuando han sido referidas en estudios de la anelidofauna para el Golfo de México, Venezuela, Brasil y otras áreas del Caribe, pueden ser consideradas como cuestionables, aun cuando las características de éstas coincidan con lo señalado en las claves y trabajos regionales. Es por ello que se hace necesario realizar un mayor número de estudios que permitan clarificar la distribución de tales especies.

\section{RESUMEN}

Se recolectaron poliquetos epibiontes de ostiones (Spondylus americanus Hermann, 1781) recolectados entre 10 y $30 \mathrm{~m}$ de profundidad en el Parque Nacional Mochima, Venezuela, empleando equipo de buceo autónomo. Se analizaron 32 ostiones, identificándose 43 especies de poliquetos; $51.3 \%$ del Atlántico, 28.2\% cosmopolitas, 17.9\% anfiamericanas y $2.6 \%$ "disyuntas".

Palabras clave: poliquetos, Spondylus americanus, bivalvos, Venezuela.

\section{REFERENCIAS}

Abbott, R.T. 1974. American seashells. Van Nostrand Reinhold. Nueva York, EEUU. 663 p.

Basilio, C.D., J.I. Cañete \& N. Rozbaczylo. 1995. Polydora sp. (Spionidae) un poliqueto perforador de las valvas del ostión Argopecten purpuratus (Bivalvia: Pectinidae) en Bahía Tongoy, Chile. Rev. Biol. Mar. 30: 71-77.

Bastida-Zavala, R. \& H. ten Hove. 2002. Revision of Hydroides Gunnerus, 1768 (Polychaeta: Serpulidae) from the western Atlantic region. Beaufortia. Zool. Mus. Univ. Amsterdam. 52: 103-178.

Blake, J.A. \& J.W. Evans. 1973. Polydora and related genera as borers in mollusk shells and others calcareous substrates (Polychaeta: Spionidae). Veliger. 15: 235-249.

Cáceres-Martínez, J. 2001. Parasitología en moluscos pectínidos, p. 343-356. In A.N. Maeda-Martínez (ed.). Los Moluscos Pectínidos de Iberoamérica. Ciencia y Acuicultura, México, DF.

Ciocco, N.F. 1990. Infestación de la viera Tehuelche (Chlamys tehuelcha (D'Orbigny)) por Polydora websteri Hartman (Polychaeta: Spionidae) en el Golfo de San José, (Chubut, Argentina): un enfoque cuantitativo. Biol. Pesq. 19: 9-18.

De León González, J.A., A. Leija-Tristán \& S. SalazarVallejo. 1993. Epifauna del ostión espinoso Spondylus princeps unicolor (Mollusca: Bivalvia) de Puerto Escondido, Golfo de California, México. Rev. Biol. Trop. 41: 877-881.

Díaz, O. 1999. Poliquetos asociados a sustratos artificiales sumergidos en la costa nororiental de Venezuela. Tesis MSc., Universidad de Oriente, Cumaná, Venezuela. $166 \mathrm{p}$.

Díaz, O. \& I. Liñero-Arana. 2003a. Poliquetos epibiontes de Pinctada imbricata Röding, 1758 (Bivalvia: Pteriidae) en el Golfo de Cariaco, Venezuela. Interciencia 28: 298-301.

Díaz, O. \& I. Liñero-Arana. 2003b. Poliquetos asociados a Isognomun alatus (Gmelin, 1791) (Bivalvia: Isognomonidae) en la costa nororiental de Venezuela. Iberus 21: 61-65.

Díaz, J.M. \& M. Puyana. 1994. Moluscos del Caribe Colombiano. Un catálogo ilustrado. COLCIENCIAS, Fundación Natura e INVEMAR, Bogotá, Colombia. $367 \mathrm{p}$. 
Humfrey, M. 1975. Sea shells of the West Indies: A guide to the marine molluscs of the Caribbean. William Collins, Glasgow, Escocia, Reino Unido. 351 p.

Keen, A.M. 1971. Sea Shells of Tropical West America. Stanford Univ. Stanford, California, EEUU. 1064 p.

Keough, M. 1984. Dynamics of the epifauna of the bivalve Pinna bicolor: interaction among recruitment, predation and competition. Ecology 63: 677-688.

Liñero-Arana, I. 1996. Aspectos bioecológicos de los poliquetos y descripciones de algunas especies bénticas de la costa noroiental de Venezuela. Trab. Asc.
Inst. Oceanogr. Venezuela, Universidad de Oriente, Cumaná, Venezuela. 254 p.

Liñero-Arana, I. 1999. Poliquetos (Annelida: Polychaeta) asociados al mejillón verde Perna viridis en la Península de Araya, Venezuela. Bol. Inst. Oceanogr. Venezuela. Univ. Oriente, Cumaná. 38: 53-62.

Perkins, T. 1984. Revisión de Demonax Kinberg, Hypsicomus Grube and Notaulax Tauber, with a review of Magelomma Johansson from Florida (Polychaeta: Sabellidae). Proc. Biol. Soc. Wash. 97: 285-368.

Widman, J.C. \& E.W. Rhodes. 1991. Nursery culture of the bay scallops, Argopecten irradians irradians in suspended mesh nets. Aquaculture 99: 257-267. 